$$
\text { Conf- } 920311--13
$$

GA-A20903

\title{
IMPURITY PENETRATION MAY 261992 AND TRANSPORT DURING VH-MODE ON DIII-D
}

\author{
by \\ S.I. LIPPMANN, T.E. EVANS, G.L. JACKSON \\ and W.P. WEST
}

MAY 1992

\section{GEMERAL ATOMICS}




\section{DISCLAIMER}

This report was prepared as an account of work sponsored by an agency of the United States Government. Neither the United States Government nor any agency thereof, nor any of their ernployees, makes any warranty, express or implied, or assumes any legal liability or responsibility for the accuracy, completenesa, or usefulness of any information, apparatus, product, or process disclosed, or represents that its use would not infringe privately owned rights. Reference herein to any specific commercial product, process, or service by trade name, trademark, manufacturer, or otherwise, does not necessarily constitute or imply its endorsement, recommendation, or favoring by the United States Government or any agency thereof. The views and opinions of authors expressed herein do not necessarily state or reflect those of the United States Government or any agency thereof. 


\title{
IMPURITY PENETRATION AND TRANSPORT DURING VH-MODE ON DIII-D
}

\author{
by \\ S.I. LIPPMANN, T.E. EVANS, G.L. JACKSON \\ and W.P. WEST
}

This is a preprint of a paper to be presented at the 10th International Conference on Plasma Surface Interactions, March 30 through April 3, 1992, Monterey, California, and to be printed in the Proceedings.

Work supported by

U.S. Department of Energy

Contract No. DE-AC03-89ER51114

GENERAL ATOMICS PROJECT 3466

MAY 1992

\section{GENERAL

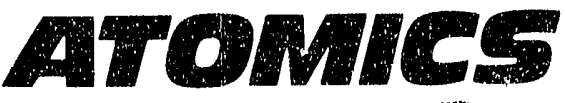 \\ (1) \\ Hed}

DISTRIBUTION OF THIS ODCUMEETT IS UNTIMTER 


\begin{abstract}
A new modeling effort is made in order to understand the observed relatively low levels of impurity contamination during the VH-mode phase on DIII-D, as compared to those observed during the $\mathrm{H}$-mode phase of selected discharges. The key element is the inclusion of the real 2-D flux surface geometry in the prediction of impurity penetration of sputtered atoms through the scrape off layer into the core plasma. Of the elements which determine the impurity content in the plasma: sputtering yield, penetration, and core transport, the penetration through the scrape-off layer is found to be the most determinative factor. The low impurity content in $\mathrm{VH}$-mode is attributed to the development of a scrape-off layer with higher density and temperature properties than those normally obtained in H-rnode.
\end{abstract}




\section{INTRODUCTION}

The improvement in $\tau_{E}$ by a factor of 1.5-2 over H-mode on DIII-D was achieved after the first boronization in May 1991, and is now routinely observed under suitably clean wall conditions. A clear effect of boronization, which may be indirectly responsible for this improvement in confinement, is the overall reduction of the levels of all of the intrinsic impurities, beginning with the ohmic phase of the discharge. The reduction of edge radiation leads to hotter edge temperatures which continue to increase during the $\mathrm{VH}$-mode. During the VH-mode phase, impurities generally do not accumulate in the center of the discharge as they do in ELM-free H-modes. This lack of accumulation is the result of some combination of three factors: (1) a reduction in the source of sputtered impurities at the surfaces, (2) changes in the bulk transport behavior, and/or (3) a decrease in the efficiency of penetration of impurities through the separatrix as a consequence of the increased $T_{e}$ and or $n_{e}$ in the scrape-off layer (SOL).

It is assumed that the source of core impurities most sensitive to edge conditions is wall sputtering by fast charge exchange neutrals, rather than from particles sputtered by the plasma at the strike points, since (as confirmed below) the latter ionize readily in the divertor region and are then governed by the plasma transport equations. The residual amount of impurities in the plasma during the period of maximum wall screening can be attributed to the these particles originating at the divertor. The source of nickel is determined by the flux and spatial and energy distributions of fast neutral deuterium atoms incident on the wall. As discussed elsewhere in these proceedings [1], this flux is roughly a factor of two smaller in VH-mode compared to H-modeSince the impurity confinement times, as measured from copper bursts, are longer by the same factor, the product of the fast neutral flux and impurity confinement times does not differ greatly between VHand $\mathrm{H}$-modes, leaving the third factor mentioned above, penetration efficiency, a strong 
candidate to account for the large observed difference in impurity accumulation between the two modes. 


\section{PENETRATION MODEL}

Figure 1 shows the 2-D geometry for shot 73182 at $t=2300 \mathrm{~ms}$. The polygonal boundary represents the plasma facing components of the interior of DIII-D. The curved lines represent a convenient family of flux surfaces generated with the output of the EFIT magnetics fitting code for this timeslice [2]. The $x$ 's represent the position of the 40 Thomson scattering points which are obtained every $25 \mathrm{~ms}$ throughout the discharge [3]. The fan of lines represents the angular distribution of particles sputtered from a given point on the wall which have a direct path to the separatrix. For each chosen timeslice, a flux surface geometry is generated, and the $T_{e}$ and $n_{e}$ associated with each surface is determined by interpolation of the Thomson scattering data. The electron temperature and density will be assumed to be constant on the flux surfaces. The double-null nature of the discharge precludes extrapolation of the scrape-off parameters from outer to inner flux surfaces, but for simplicity it will be assumed that $\lambda_{i n}=\lambda_{o u t}$. In any case, most attention here will be placed on the ability of the outer scrape-off layer to shield nickel coming from the outer wall.

The VH-mode discharges considered here were obtained under boronized wall conditions. The underlying surfaces are continusus carbon tiles on the top, bottom and centerpost, and the largely exposed inconel vacuum vessel walls on the five outer wall poloidal segments. Boronization reduces the intrinsic carbon and oxygen levels by a factor of 3-7 and the nickel level by a factor of 5-20 in the ohmic phase, with general but more varying reductions in auxiliary-heated phases [5]. The boronization layer is known to be uneven, and some effective fractional area of the walls is the source of impurities other than boron. It is assumed that the nickel comes from the outer wall, carbon from the carbon tiles, and oxygen and boron from all of the surfaces. 


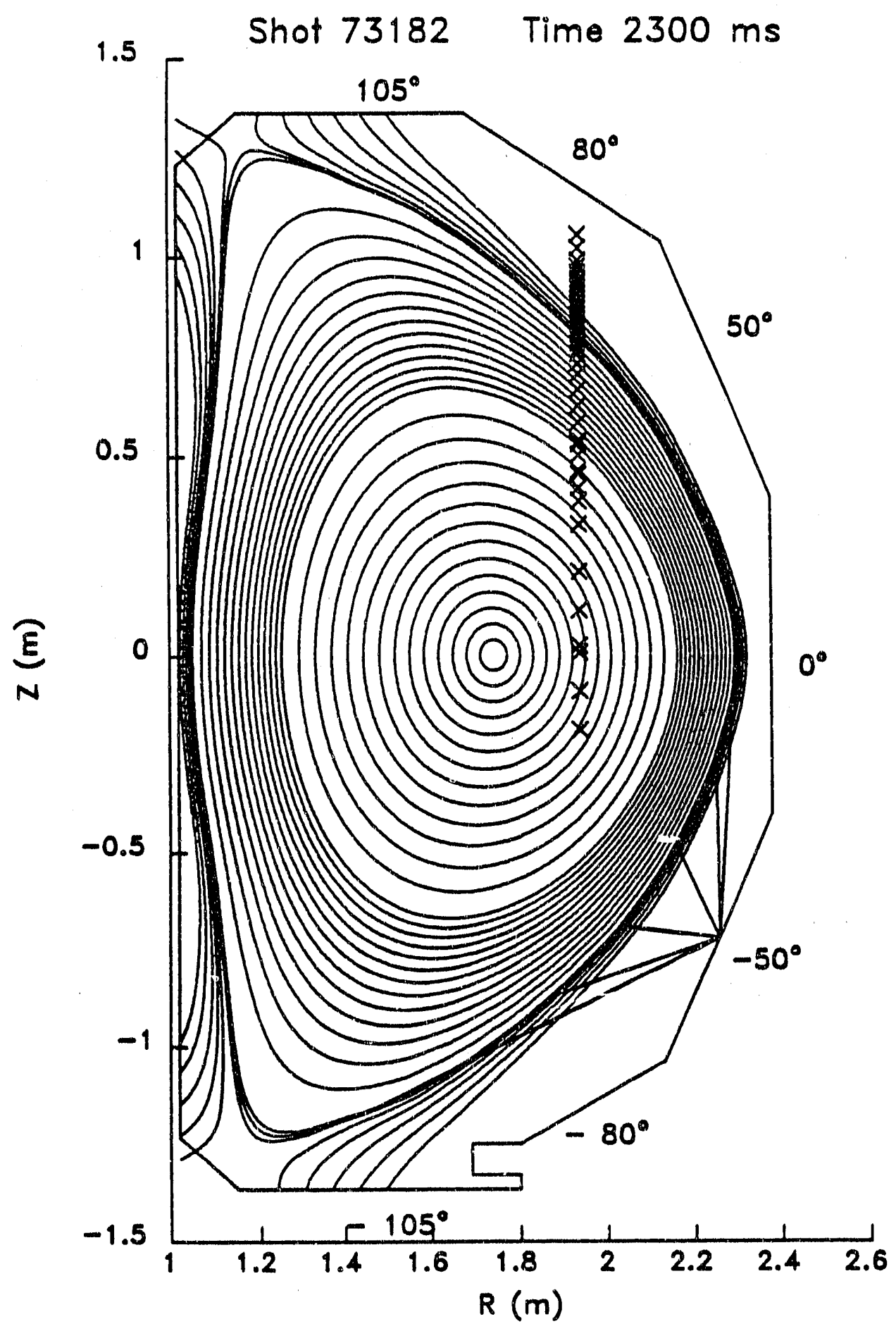

Fig. 1. Cross-section of of shot 73182 at $2300 \mathrm{~ms}$, showing flux surfaces, physical boundaries, Thomson scattering points, and the location of the 12 test points for penetration. 
The energy distribution of the sputtered particles does affect the penetration probability. Data is scarce on this subject, so the initial model relies on the published data for gold sputtered by deuterium [6]. The angular distribution is also dependent on the distribution of incident fast neutrals, so the distribution is assumed to be $\cos ^{1.0} \theta$ for $\operatorname{sim}$ plicity. Since the incident particles are generally directed outward, the power of the cosine is probably more than 1.0, but this is offset by the fact that the 2-D model is being used to approximate the 3-D situation, in which sputtered particle motions have a toroidal component.

In the model developed here, the sputtered particles are launched from the walls toward the separatrix, and those which ionized before reaching the separatrix are considered to be prevented from penetrating, although as mentioned above, the divertor is expected to contribute a fixed amount to the total core impurity level. The ionization rates used are taken from reference [7]. As mentioned in the previous section, impurity confinement times are found to be close to the energy confinement time, making it unnecessary to model the core transport in detail for the present purpose, which is mainly to show that the penetration model, even with all the assumptions made in this section, can explain the relative lack of impurity accumulation in VH-mode when compared to that found in $\mathrm{H}$-mode. 


\section{EXPERIMENTAL RESULTS}

Figures 2 and 3 show the temporal evolution of intrinsic impurity lines and other relevant parameters during two shots containing VH-mode phases. Shown are interferometric density, $D_{\alpha}$, carbon, oxygen, and nickel signals from the SPRED spectrometer, the energy confinement time, $\tau_{E}$ (obtained from the plasma diamagnetism), and the injected and radiated power. Figure 2 shows the evolution of the good VH-mode shot whose flux surfaces are depicted in Fig. 1. It has a long $(600 \mathrm{~ms})$ VH-mode phase shortly after the start of the beams. At $2700 \mathrm{~ms}$ it becomes an ELMing $\mathrm{H}$-mode, and as the beam power is lowered, the ELMs become less frequent and eventually stop, leading to impurity accumulation and eventual radiative collapse of the discharge. In the VH-phase of this discharge, the impurities are effectively screened, helping to sustain the VH-mode. In contrast, shown in Fig. 3, is a similar discharge with short lived VH-mode. It is not clear why this VH-modedoes not develop a thick scrape-off layer, but it may be related to the fact that one neutral beam is turned on early, enabling the impurity levels to rise in L-mode. In any case, they begin to rise further at the start of the VH-mode, and the plasma reverts to a ELM-free $\mathrm{H}$-mode after about $100 \mathrm{~ms}$. This $\mathrm{H}$-mode is unusual in that a thick scrape-off layer develops and causes the impurity level to drop, whereas in the usual ELM-free H-mode, impurity accumulation would take place.

Before asing the new penetration model to explain the different behavior of the different phases and shots, it is important to consider changes in bulk impurity confinement, to demonstrate that changes in confinement cannot account for the observed behavior. A convenient measure of impurity confinement on DIII-D is prorided by bits of copper, from the copper foam tile backing, falling into the discharge at random intervals. The discontinuous nature of the copper burst allows a simple measure of confinement time by measuring the decay time of $\mathrm{Cu}$ XXVI. Figure 4 shows the results from analysis of a 


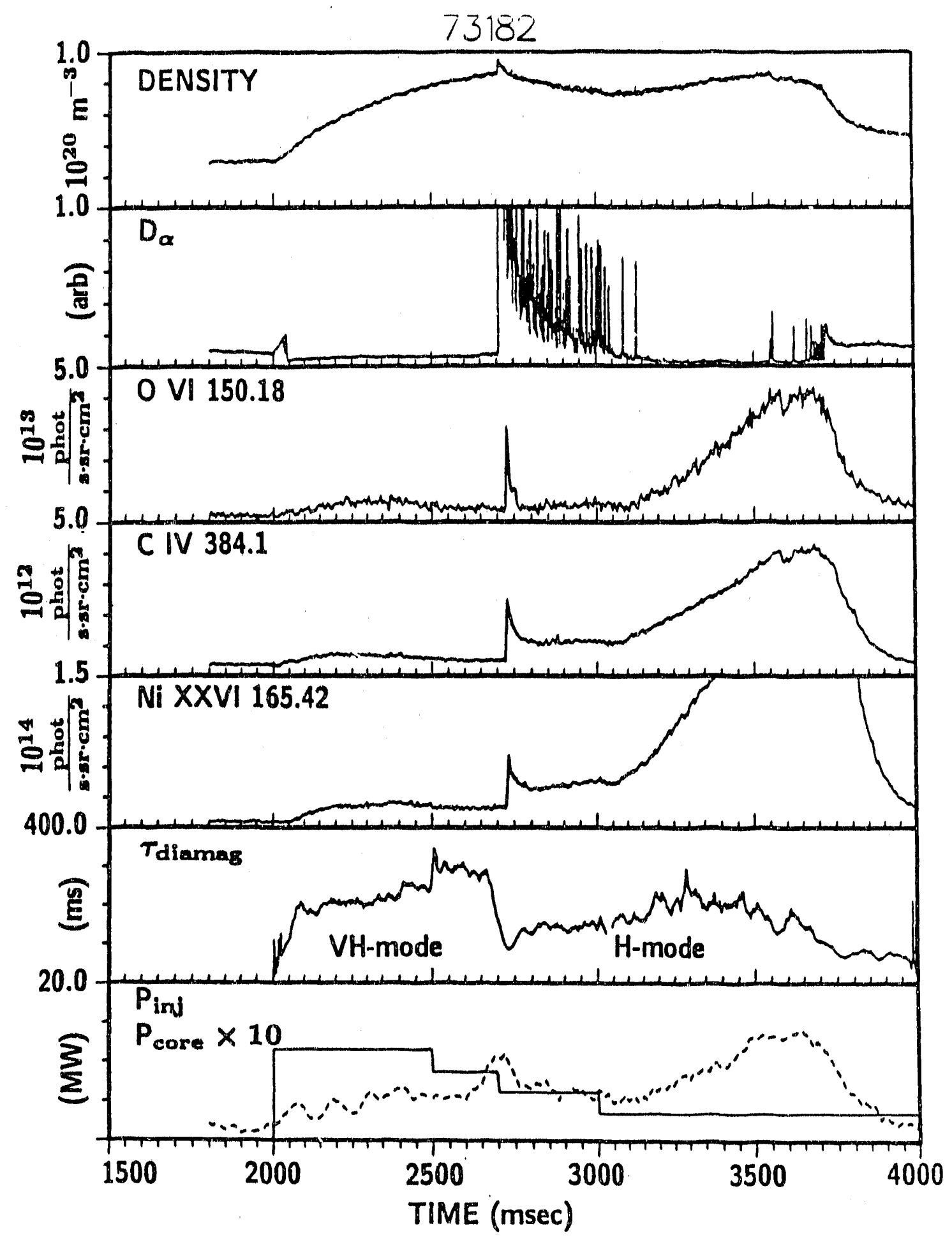

Fig. 2. Parameters during Shot 73182: interferometric measured $n_{e}, D_{\alpha}$, SPRED signals, $\tau_{E}$, and the injected and radiated power. $\mathrm{VH}$-mode and $\mathrm{H}$-mode phases are indicated. 


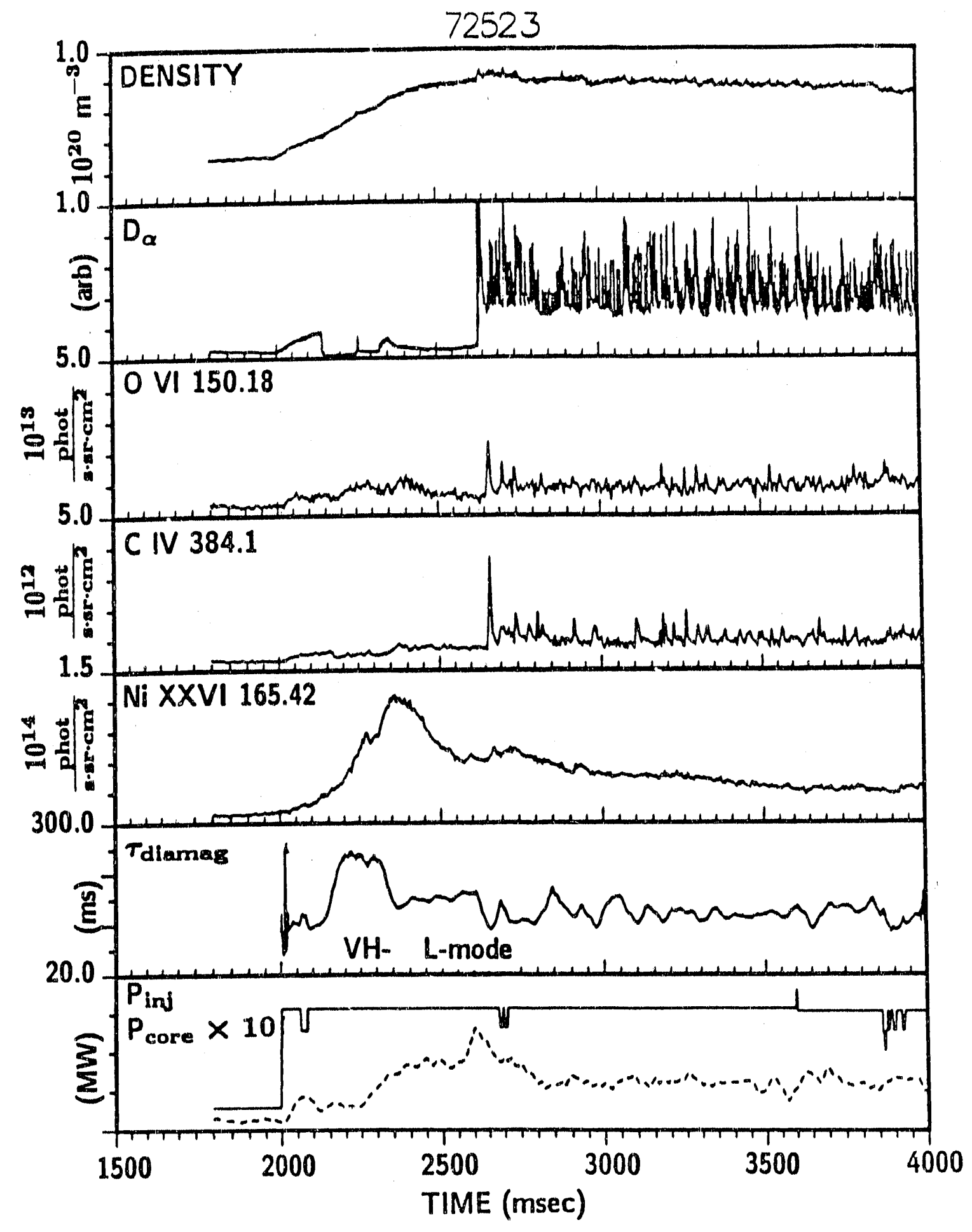

Fig. 3. Parameters during Shot 72523: interferometric measured $n_{e}, D_{\alpha}$, SPRED signals, $\tau_{E}$, and the injected and radiated power. $\mathrm{VH}$-mode and $\mathrm{H}$-mode phases are indirated. 


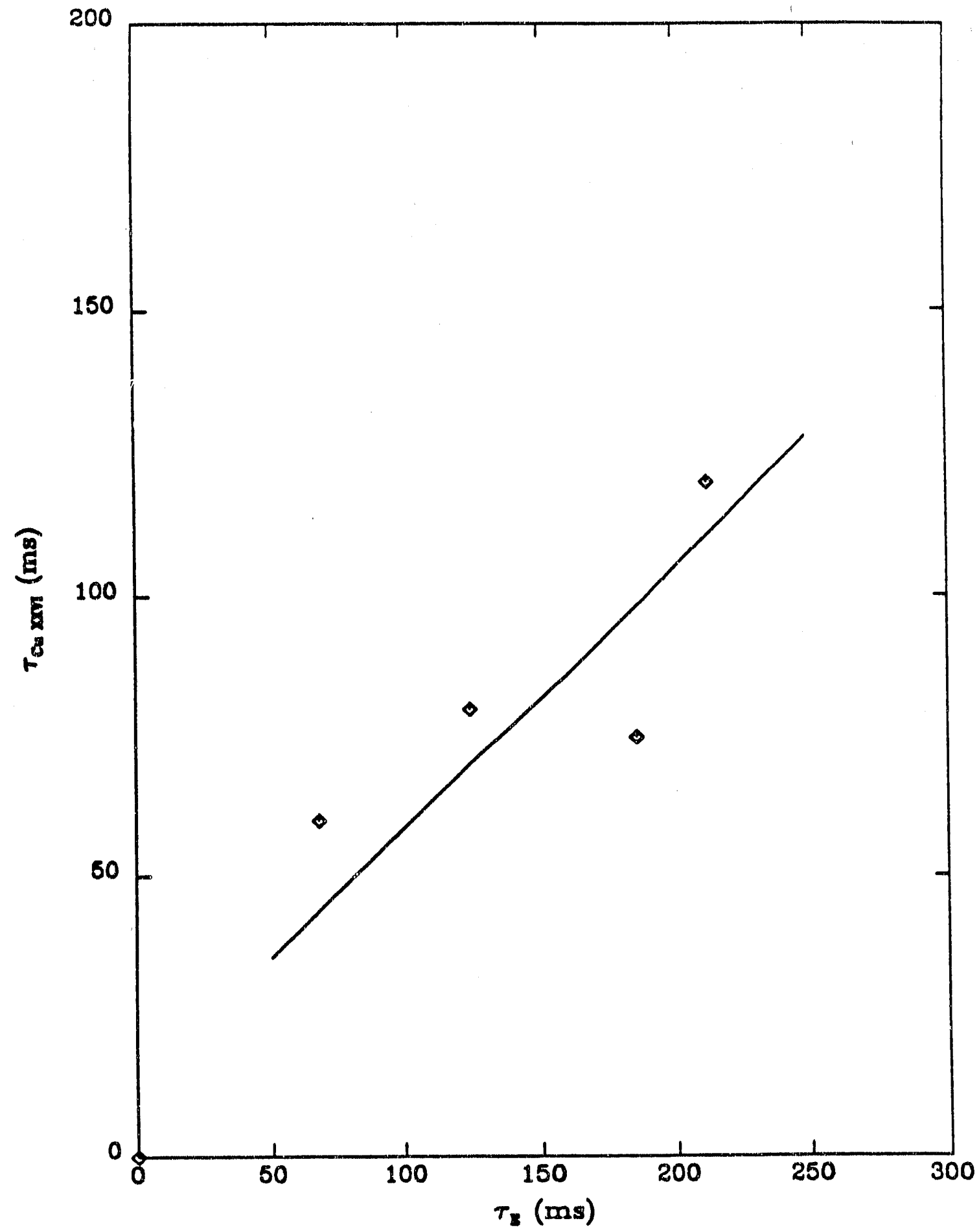

Fig. 4. Copper $X X V I I=$ line brightess decay time, indicative of impurity confinement time vs energy confinement time. 
series of copper bursts into VH- and H-mode phases. A near unitary relation is found between energy confinement time, $\tau_{E}$, and copper confinement time, $\tau_{\mathrm{Cu}} \mathrm{XxVI}$. For a given discharge, such as 73182, the longer confinement time in VH-mode would tend to increase the impurity levels compared to those in the later $\mathrm{H}$-mode, but the opposite is observed. The decrease in neutral fueling and increased confinement time in VH-mode relative to H-mode tend to cancel each other out. Therefore, they are not a significant factor when compared to the dramatically different penetration rates predicted by the new model. 


\section{RESULTS OF THE PENETRATION MODEL}

Figures 5 and 6 show the results of the penetration model for carbon, oxygen and nickel applied to the two test discharges. Shown in the upper portion of the figures are time sequences of the scrape-off $T_{e}$ and $n_{e}$ profiles between $1.0 \leq \rho \leq 1.1$, determined by mapping the Thomson scattering data to the scrape-off flux surfaces where $\rho=\psi-$

$\psi_{\text {axis }} / \psi_{\text {sep }}-\psi_{\text {axis }}$ with the $\psi$ 's being poloidal flux at the magnetic axis and separatrix. The VH-mode, ELMing axd ELM-free H-mode phases are indicated. Below are the predicted penetration fractions for the sputtered intrinsic impurities. Note that in the VH-phase of shot 73182 , there develops a very hot and dense scrape-off plasm:n, and that the penetration fraction under these conditions becomes quite small. When the VH-mode phase ends, most of the scrape-off layer disappears, and the efficiency of penetration increases by about a factor of 20. As long as ELMs are present, impurities are kept under control, since the impurities in the outer portion of the discharge are removed at each ELM. However, in the low-power, ELM-free phase, the impurities accumulate, and continue to rise until a point of radiative collapse, since the equilibrium concentrations for the new level of impurity influx is apparently bigher than the radiative threshold for collapse.

Figure 6 shows the penetration probability for carbon, oxygen and nickel as a function of poloidal position for shot 73182 at $2300 \mathrm{~ms}$. It is noted that the predicted penetrating fraction of particles sputtered by fast neutrals from the floor and ceiling is very small. This helps to justify the assumption that the source of impurities from the rest of the wall is the dominant one, rather than that from plasma sputtered particles near the strike points.

Figure 7 shows the result of the modei for shot 72523. In this case, a hot and dense VH-mode scrape-off layer does not develop, and the penetration efficiency remains high. The impurity levels rise, and this rise may be responsible for the short duration of the $\mathrm{VH}$-mode, and reversion to $\mathrm{L}_{1}-$ mode. 

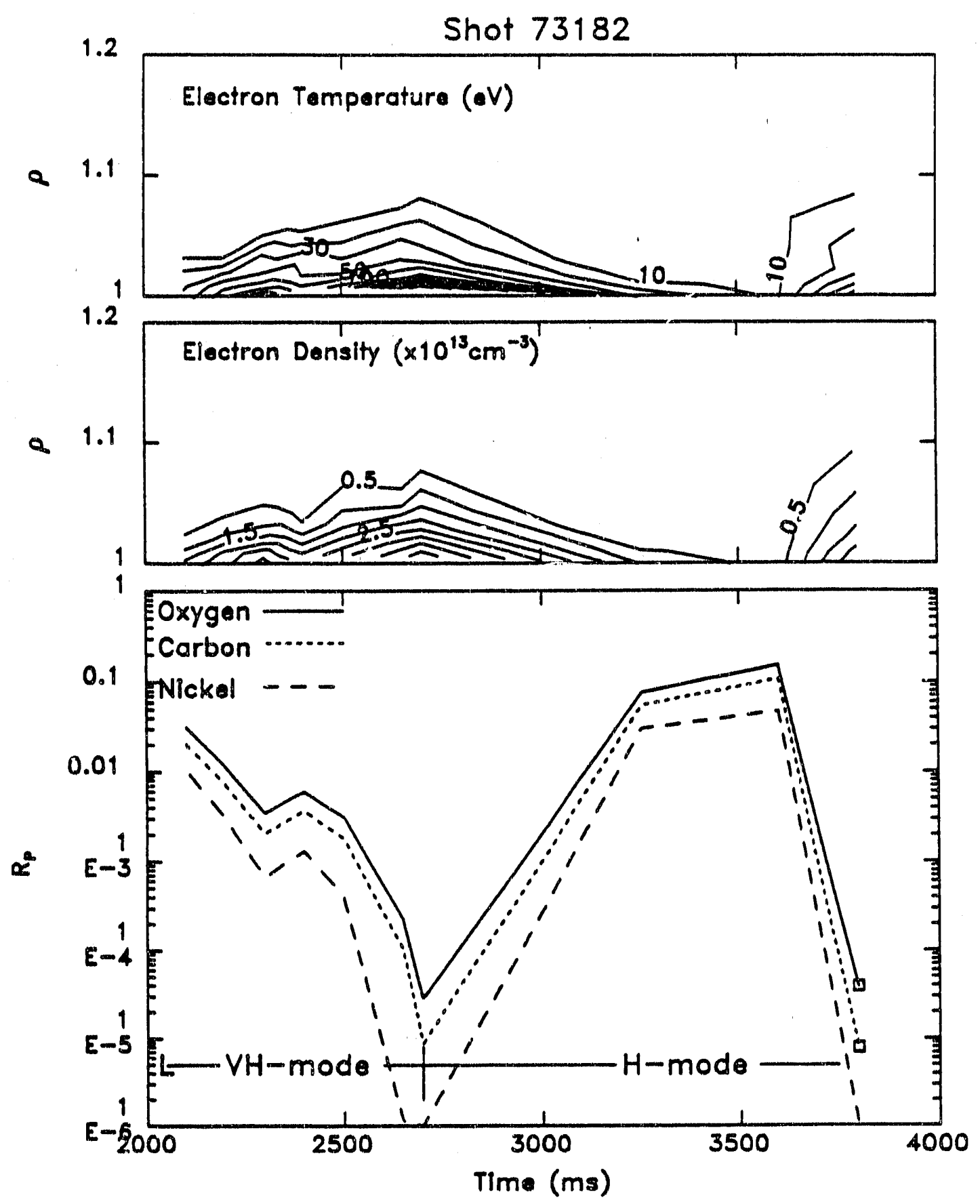

Fig. 5. Shot 73186: good VH-mode case. Contour plot of scrape-off $T_{e}$ and $n_{e}$ as a function of effective radial localities $r / a=\rho$ and time, along with the results of the 2-D penetration model for carbon, oxygen and nickel, showing the percentage if launched neutral $C, O$, $\mathrm{Ni}$ particles that make it past the separatrix. 


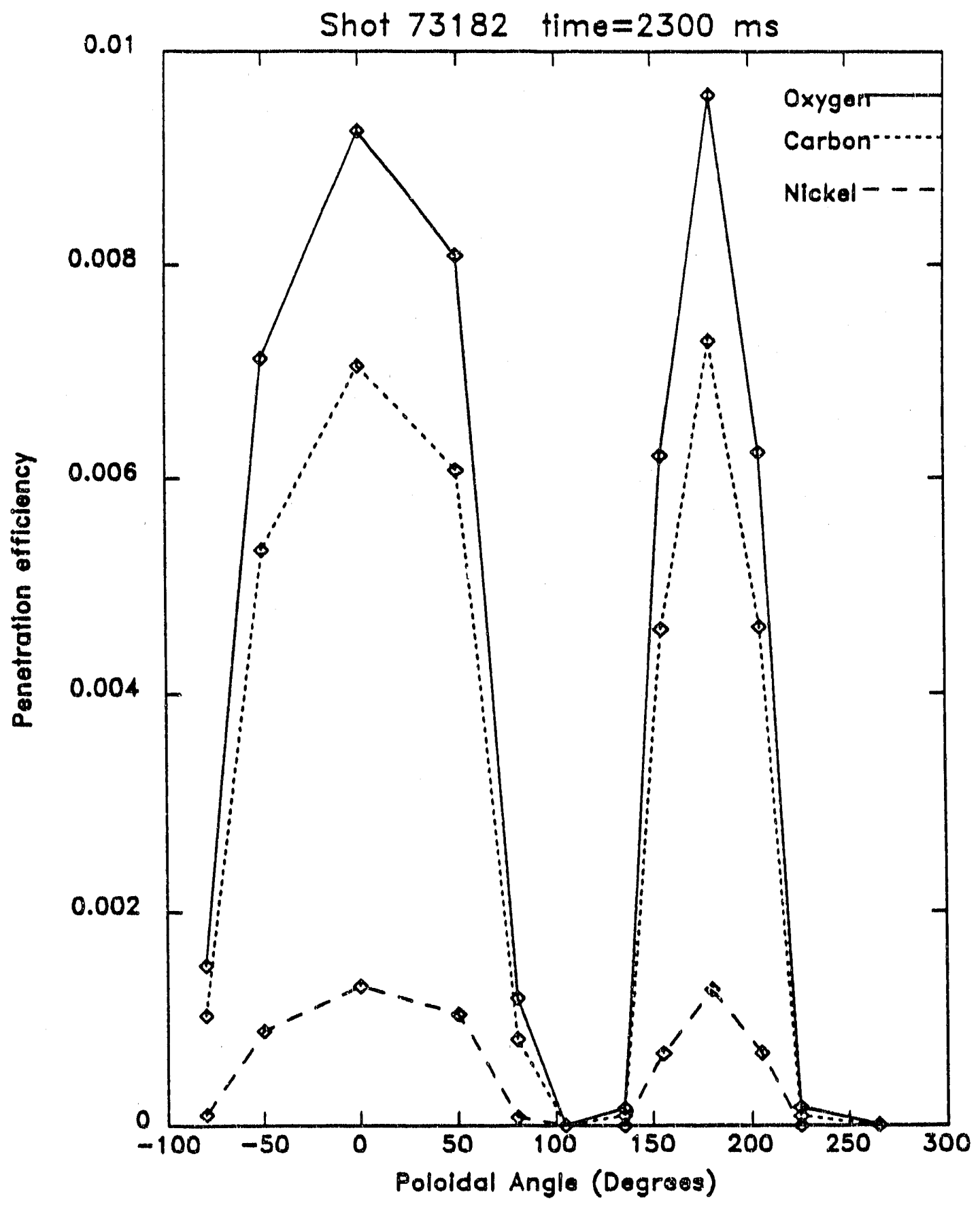

Fig. 6. Penecration probability for carbon, oxygen and nickel as a function of poloidal position for shot 73182 at $2300 \mathrm{~ms} .0^{\circ}$ is at the outer midplane, $110^{\circ}$ the ceiling, $180^{\circ}$ the inner midplane, and $260^{\circ}$ the floor, as indicated in Fig. 1. 

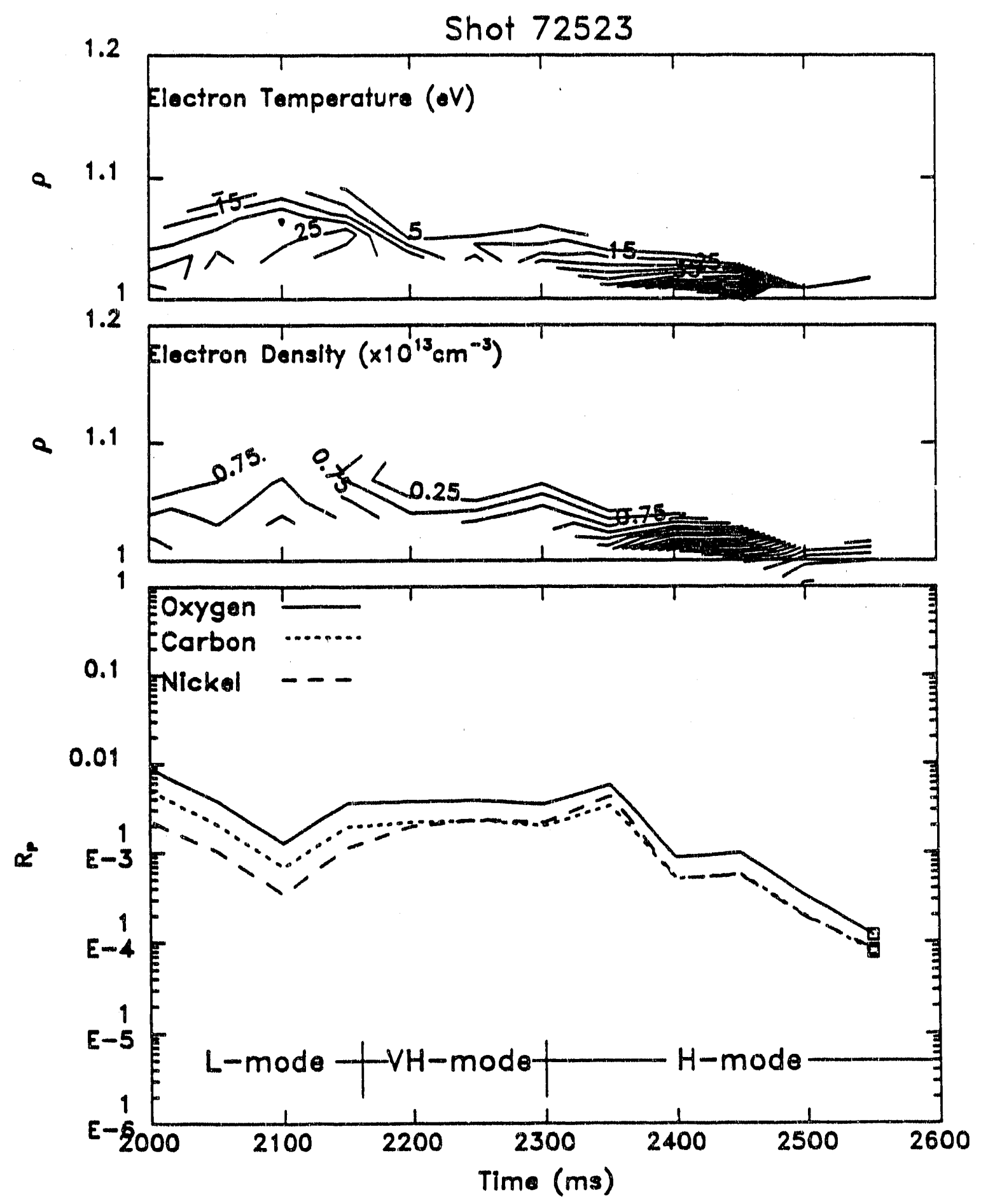

Fig. 7. Shot 72523: marginal VH-mode. Contour plot of scrape-off $T_{e}$ and $n_{\mathrm{c}}$ as a function of $\rho$ and time, along with the results of the 2-D penetration model for carbon, oxygen and nickel. 


\section{DISCUSSION}

The penetration model clearly demonstrates the importance of maintaining a sufficient scrape-off layer in the double-null configuration to prevent the accumulation of intrinsic impurities sputtered by fast neutrals from the walls. More work needs to be done to determine what makes the scrape-off plasma more robust (hotter and denser) in shot 73182. Careful consideration will be given to the exact details of the magnetic configuration for a series of $\mathrm{VH}$-mode discharges, in an attempt to relate the scrape-off thickness to such things as inner and outer gaps, $\mathrm{X}$-point heights, and degree of DND balance. In addition, things which might lengthen the traditional scrape-off layer decay length, $\lambda=\sqrt{\left(D_{\perp} L_{\|} / v_{\triangleleft}\right)}$, could be tried in future experiments, sich as increasing $D_{\perp}$ by ergodizing scrape-off flux surfaces, and gas puffing to cool the scrape-off plasma and reduce $v_{s}$, while at the same time increasing edge $n_{e}$. There is already evidence from DIII-D radiative divertor experiments that gas puffing leads to a cleaner plasma [8].

It should also be noted that the pre-VH-- L-mode scrape-off layer is found to be more robust than in pre- $\mathrm{H}$ and $\mathrm{L}$-mode. In light of the present results, it seems clear that this would keep the L-mode cleaner, which would keep the edge less radiative and therefore hotter, increasing the edge current, and influencing the development of the discharge in a fundamental way.

To make the penetration model more quantitative, better data is needed for sputtering yield, energy and angular distribution. With this data, the present model could be coupled with DEGAS for fast neutral flux. This improved model could then be coupled with other impurity transport codes for inclusion of plasma transport impurity sources from the divertor, and a MIST-like core impurity transport code, to make a quantitative prediction for impurity concentration for comparison with the experimentally determined values on DIII-D. 
This work was supported by the U.S. Department of Energy under Contract No. DE-AC03-89ER51114. 


\section{REFERENCES}

[1] G.L. Jackson et al. "Boronization in DIII-D," this conference.

[2] L.L. Lao et al. Nucl. Fusion 30 (1990) 6.

[3] T.N. Carlstrom et al. "Design and Operation of the Multipulse Thomson Scattering Diagnostic on DIII-D," to be published in Rev. Sci. Instrum.

[4] M.F.A. Harrison et al. Nucl Technol./Fusion 3 (1983) 432.

[5] W.P. West et al. Bull. Am. Phys. Society, 36 (1991) 2476 (Abstract).

[6] P. Hucks et al., J. Nucl. Mater. 76 \& 77 (1978) 136.

[7] J. Shull and M. VanSteenburg, Ap. J. 48 (1987) 95.

[8] T.W. Petrie et al. Proceedings of the 18th European Conference on Controlled Fusion and Plasma Physics, Berlin, Germany (1991), Vol. III, p. 273. 

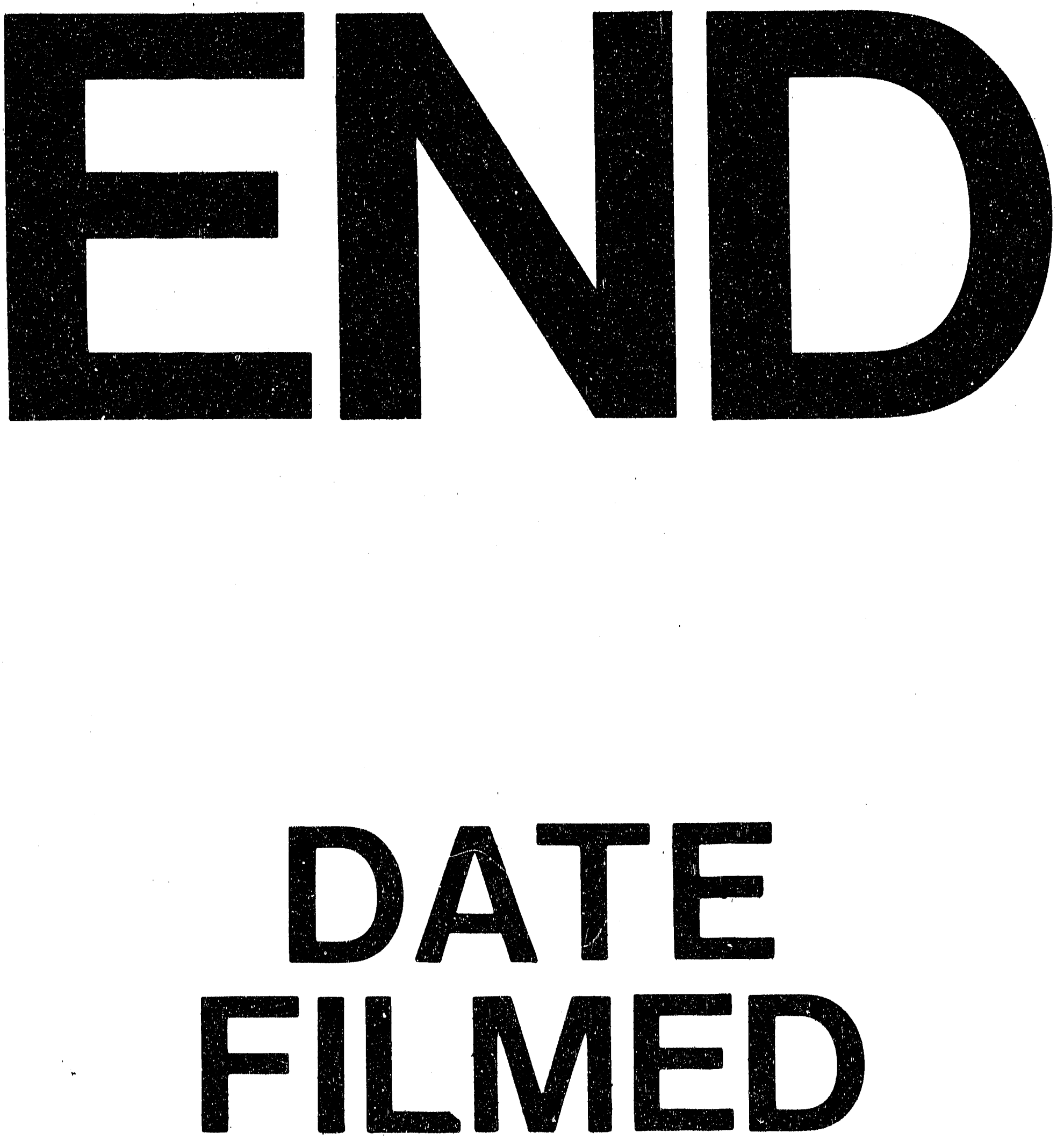

1

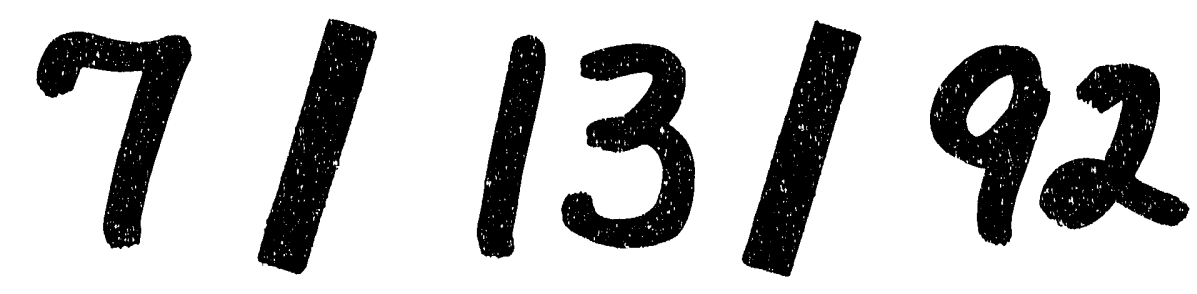


